\title{
Experiencia de evaluación por pares usando retornables en el Grado de Ingeniería Informática (UPV)
}

\author{
María Teresa Gassó Matoses ${ }^{\mathrm{a}}$, Antonio Martí-Campoy ${ }^{\mathrm{b}}$, Vicente Atienza Vanacloig \\ Salvador Petit Martí y Franciso Rodríguez-Ballester ${ }^{\mathrm{b}}$ \\ ${ }^{a}$ Departamento Matematica Aplicada, Universitat Politecnica Valencia, Camino de Vera s/n, 460021 \\ Valencia. mgasso@mat.upv.es, ${ }^{\mathrm{b}}$ Departamento Disca, Universitat Politécnica Valencia, Camino de \\ Veras/n 46021 Valencia. \{amarti, spetit,vatienza, prodrig\}@disca.upv.es.
}

\begin{abstract}
This work present the results of a peer assessment experiment performed with a learning tool named "returnable" in the subjects "Algebra" and "Computer Fundamentals" from the Computer Engineering degree that is conducted at Universitat Politècnica de València (UPV). A returnable is a set of exercises that are similar to those that can be found in a conventional exam and that are carried out after the corresponding topic has been taught. The main benefit of the tool comes from its usage in a peer assessment context since the feedback to the students is delivered earlier. Moreover, it allows the development of the high-level skills that are necessary to analyze and evaluate other student returnables.
\end{abstract}

The main goal of the experiment is to validate the marks that the students obtain from their peers. The validation will increase the usefulness of the returnable tool as a feedback mechanism and for the overall course evaluation.

Keywords: continuous assessment, peer assessment, computer engineering degree.

\footnotetext{
Resumen

En este trabajo, se presenta una experiencia de evaluación por pares utilizada con la herramienta "retornables" en las asignaturas de Álgebra y de Fundamentos de Computadores en el Grado de Ingeniería Informática. Los "retornables" son una colección de ejercicios representativos tipo examen, realizado al finalizar cada tema. Su principal interés radica en la evaluación por pares que los alumnos deben llevar a cabo. De esta forma además de tener un feedback temprano que les sirve de entrenamiento para los siguientes actos de evaluación, desarrollan habilidades de alto nivel como son analizar y evaluar el trabajo de un compañero.

El objetivo de este trabajo es determinar en qué medida es válida o no la calificación que dan los alumnos a sus compañeros. Esta validación es especialmente relevante para el alumno receptor de la calificación, y
} 
también para el profesor que quiera utilizar dicha calificación en su evaluación.

El trabajo concluye analizando los motivos que justifican estos resultados y dando pautas sobre el uso correcto que cabe dar a esta herramienta atendiendo a las pequeñas limitaciones detectadas. Este trabajo ha sido financiado por el programa Proyectos de Innovación y Mejora Educativa (PIME B05 16_17) y la ETSINF de la UPV.

Palabras clave: evaluación continua, evaluación por pares, grado en informática.

\section{Introducción y objetivos}

El nuevo modelo de Bolonia ha traído consigo la transformación de los estudios universitarios no sólo en una reestructuración de las materias, asignaturas y cursos, sino también en la forma y el momento en que los alumnos deben ser evaluados. De este modo la evaluación de una asignatura pasa de uno o unos pocos exámenes presenciales a múltiples actos de evaluación, presenciales o no, a lo largo del semestre y utilizando diferentes herramientas

Para realizar esta evaluación continua, los autores desarrollaron una herramienta docente (no informática) llamada "retornables", basada principalmente en la evaluación por pares.

La evaluación por pares forma parte fundamental de la herramienta "retornables" y ha sido utilizada por los autores desde 2010 como complemento en la evaluación en las asignaturas de Álgebra y Fundamentos de Computadores que se imparten en el grado en Ingeniería Informática de la Universitat Politècnica de València

Hay gran cantidad de trabajos relacionados con la evaluación por pares. Por ejemplo, en (Krathwohl 1964) y (Davies 1998) se postula que la evaluación y la crítica son habilidades de alto nivel en la taxonomía de Bloom y por tanto los estudiantes que participan en el proceso de revisión por pares consiguen no solo mejorar el aprendizaje de los objetivos básicos de la asignatura sino que también logran objetivos de más alto nivel. Otros muchos trabajos publicados concluyen que la evaluación por pares presenta beneficios, aunque no está exenta de inconvenientes. Uno de los problemas más importantes es la desviación en la calificación que se da a un compañero respecto a la merecida, tanto a favor como en contra. Esta desviación puede ser intencionada, por ejemplo por motivos de amiguismo o de fuerte competencia, o por desconocimiento de la materia que se está evaluando (Ibarra 2012), (Marqués 2013).

El principal objetivo del trabajo que aquí se presenta es saber hasta que punto es válida la nota asignada por los propios compañeros. Es decir, saber si un alumno puede confiar en la realimentación que recibe por parte de sus compañeros, y si el profesor puede emplearla como parte de la evaluación de la asignatura. Para alcanzar este objetivo se ha analizado la

(cc) EY-NC-ND 2018, Universitat Politècnica de València

Congreso IN-RED (2018) 
variación de la nota entre la corrección realizada por los alumnos y la corrección realizada por los profesores en los cursos 14/15, 15/16 y 16/17.

\section{Antecedentes}

De forma muy breve y para poner en contexto al lector, se describe qué es y cómo se usan los retornables.

Un retornable es una colección de ejercicios de un tema de la asignatura que los alumnos deben resolver de forma no presencial. Una vez resuelto, lo intercambian con sus compañeros para corregirlos y evaluarlos. Los alumnos disponen de criterios de corrección pero no de las soluciones. Dado que para cada tema hay dos modelos de retornable, se intenta que el alumno corrija el modelo que no ha resuelto. Finalmente el profesor registra las notas y devuelve el retornable al alumno que lo resolvió.

Una descripción detallada de qué son los retornables, cómo se usan, sus objetivos primarios y secundarios, las experiencias realizadas y el grado de satisfacción de los alumnos con esta herramienta se puede encontrar en (Martí-Campoy 2013, 2014).

\section{Análisis de la evaluación por pares}

Durante los tres cursos comprendidos entre el año 2014 y 2016 cursos los profesores han revisado y reevaluado la corrección que de los alumnos. Del total de 1102 retornables entregados, 991 fueron recorregidos por el profesor. Esto representa un $89,2 \%$ de los retornables entregados por los alumnos. Así pues en los análisis siguientes se consideran únicamente estos 991 retornables que han sido corregidos tanto por un alumno como por un profesor.

El rango de notas de cada retornable es diferente, ya que cada pregunta se puntúa entre $0 \%$ y $100 \%$ y la nota final del retornable es la suma de los porcentajes obtenidos en cada pregunta. Así, la nota máxima de un retornable variará en función del número de preguntas que contenga.

La variable que se estudía en los siguientes análisis es la diferencia entre la nota del profesor y la nota del alumno (Nota profesor - Nota alumno). Para poder comparar todos los datos recopilados, y al mismo tiempo obtener valores númericos fáciles de interpretar, esta diferencia se ha normalizado al rango $[-10,+10]$. La Tabla 1 muestra los descriptores estadísticos básicos para Nota profesor - Nota alumno, estando las notas en el rango de $0 \mathrm{a}$ 10 cómo se ha dicho anteriormente.

La Fig. 1 muestra gráficamente el porcentaje de casos en que la nota del profesor coincide, es menor, o es mayor que la nota asignada inicialmente por el alumno. En esta gráfica se puede observar que en un 35\% de los casos la corrección realizada por los alumnos es correcta y coincide con la que haría el profesor. Para aquellos casos donde el profesor y el alumno no coinciden, es mucho más frecuente que el alumno conceda una nota más elevada que la del profesor. 
Tabla 1. Descriptores estadísticos básicos para Nota profesor - Nota alumno

\begin{tabular}{lr}
\hline Descriptor & Valor \\
\hline Recuento & 991 \\
Promedio & $-0,74$ \\
Desviación Estándar & 1,59 \\
Mínimo & $-6,4$ \\
Máximo & 6,0 \\
Rango & 12,4 \\
Recuento valores $=0$ & $346(34,9 \%)$ \\
Recuento valores $<0$ & $484(48,8 \%)$ \\
Recuento valores $>0$ & $161(16,2 \%)$ \\
\hline
\end{tabular}

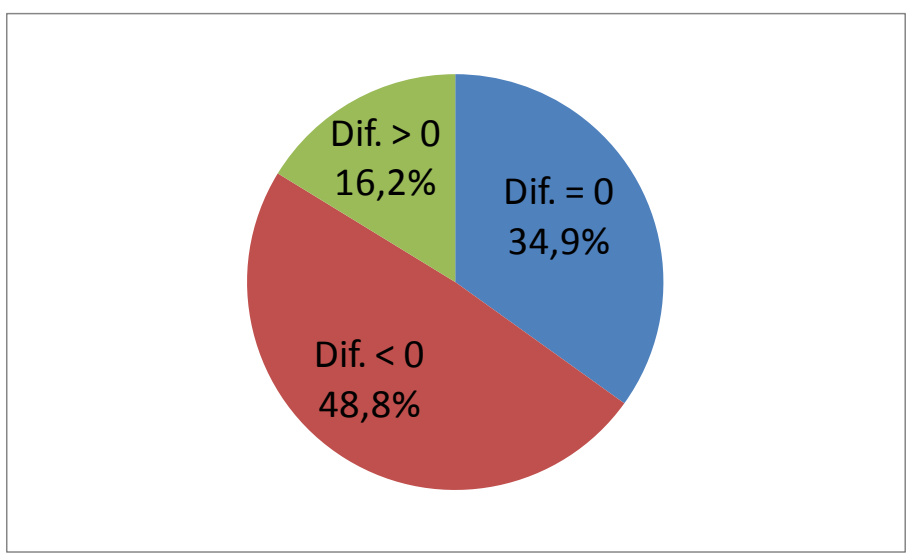

Fig 1: Grado de coincidencia entre la nota dada por el alumno corrector y la del profesor. Valores negativos indican que el alumno a dado una nota mayor que la del profesor.

Para ver con más detalle las discrepancias en la evaluación entre el profesor y los alumnos, se presenta en la Fig. 2 el histograma para Nota profesor - Nota alumno. En esta figura puede verse que la discrepancia entre la nota del profesor y la nota del alumno, tanto al alza como a la baja, se encuentra en el rango ]-1, +1 [ para el $60 \%$ de los casos. También se aprecia una tendencia hacia los valores negativos, tanto en número de casos como en magnitud. Es decir, respecto de la nota del profesor, los alumnos dan notas más altas y en más casos que en el sentido contrario.

(cc) EY-NC-ND 2018, Universitat Politècnica de València 


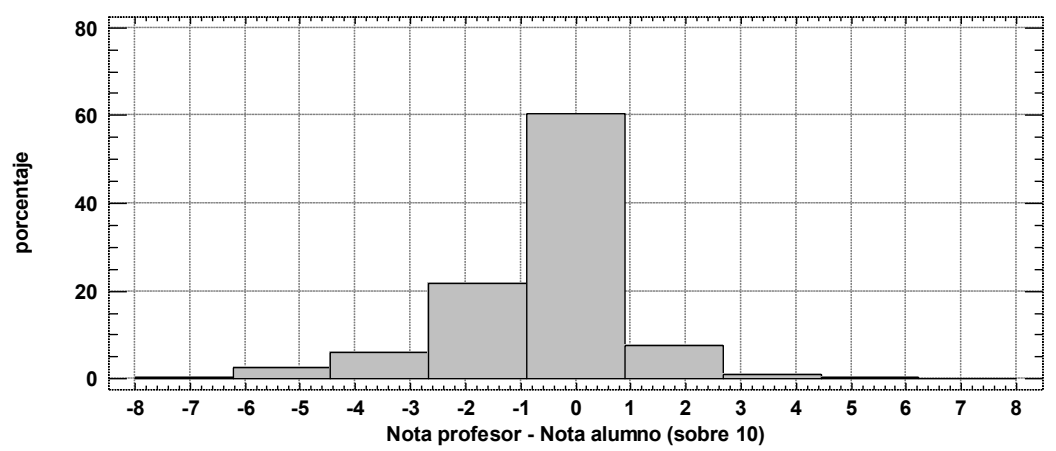

Fig 2. Histograma de frecuencia para la diferencia entre la nota del profesor y la nota del alumno, sobre 10.

La Tabla 2 muestra los principales descriptores estadísticos para la misma variable (Nota profesor - Nota alumno) separados por asignaturas. La diferencia en el recuento se debe al número de profesores, y por tanto de grupos de alumnos, que participan en la experiencia, siendo dos profesores en FCO y una profesora en Álgebra. Exceptuando esta diferencia, los valores de los descriptores son muy similares.

Tabla 2. Descriptores estadísticos básicos para Nota profesor - Nota alumno separados por asignatura

\begin{tabular}{lrr}
\hline Descriptor & Valor Álgebra & Valor Fundamentos \\
\hline Recuento & 319 & 672 \\
Promedio & $-0,7$ & $-0,5$ \\
Desviación Estándar & 1,7 & 1,4 \\
Mínimo & $-6,0$ & $-6,4$ \\
Máximo & 6,0 & 4,0 \\
Rango & 12,0 & 10,4 \\
Recuento valores $=0$ & $114(35,7 \%)$ & $232(34,5 \%)$ \\
Recuento valores $<0$ & $155(48,6 \%)$ & $329(49,0 \%)$ \\
Recuento valores $>0$ & $50(15,7 \%)$ & $111(16,5 \%)$ \\
\hline
\end{tabular}

En Fig. 3 y Fig. 4 se muestran los histogramas de frecuencia para Nota profesor - Nota alumno para la asignatura Álgebra y Fundamentos de Computadores, respectivamente. En este caso se aprecia una diferencia significativa en la distribución de la diferencia de nota. Se produce un trasvase de un $10 \%$ de los casos desde el intervalo ]-1, +1 [ hacia valores más negativos. Es decir, los alumnos de Álgebra son un poco más generosos en sus calificaciones que los de Fundamentos. Esta diferencia puede deberse a las características de las asignaturas, a los alumnos que han participado en la experiencia ya que no son los mismos en las dos asignaturas, o a la diferencia en el número de casos estudiados para cada asignatura. 


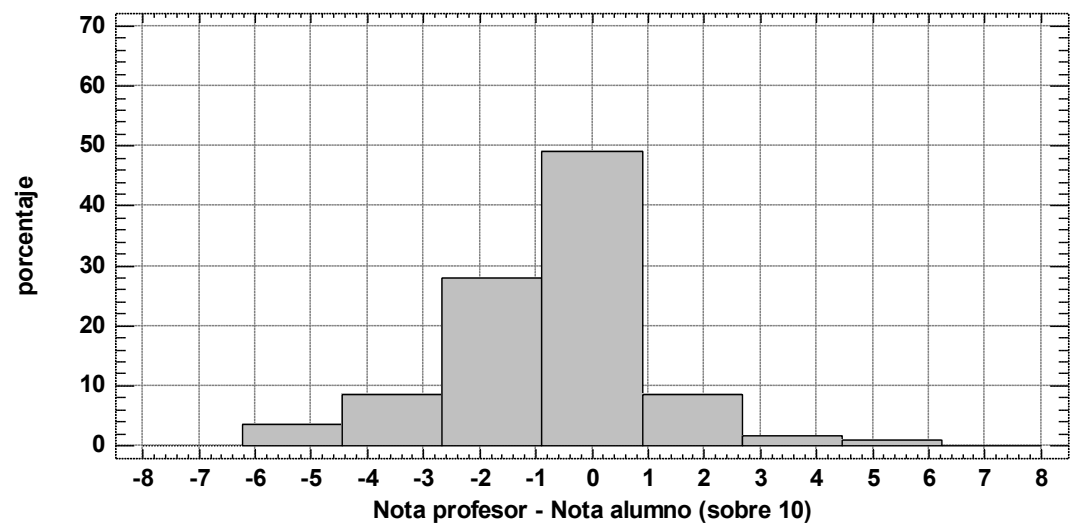

Fig 3. Histograma de frecuencia para la diferencia entre la nota del profesor y la nota del alumno, sobre 10 en la asignatura Álgebra.

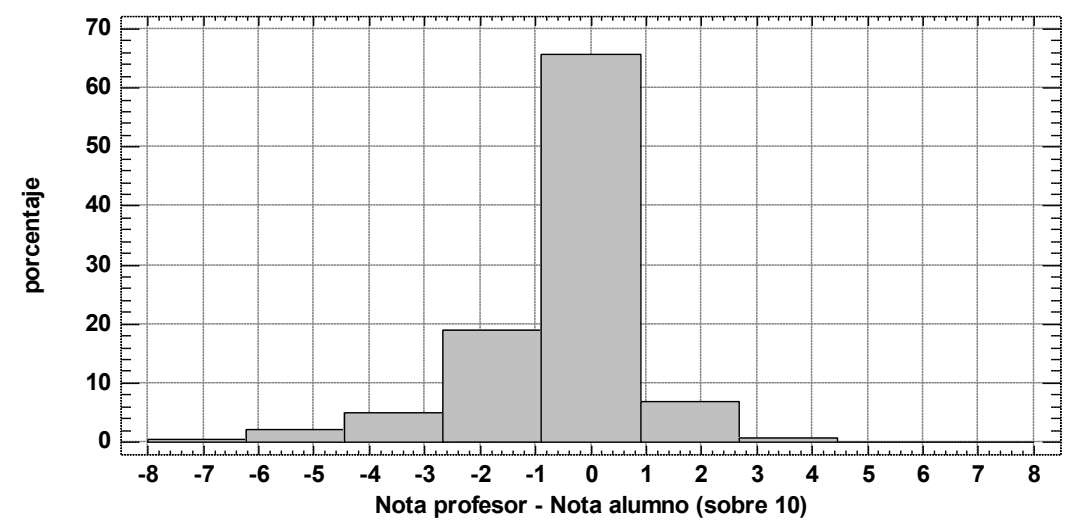

Fig 4. Histograma de frecuencia para la diferencia entre la nota del profesor y la nota del alumno, sobre 10 en la asignatura Fundamentos de Computadores.

\section{Discusión}

En primer lugar, es evidente que un $100 \%$ de concordancia entre la nota de los alumnos y de los profesores sería una anomalía, por lo que cabía esperar las desviaciones de menor magnitud que finalmente se han recogido.

En segundo lugar, en cuanto a las discrepancias de mayor magnitud, las observaciones de los profesores y comentarios de los alumnos han permitido identificar las siguientes situaciones:

Si la nota del alumno es mayor que la del profesor es debido a:

1. Alumnos que no dominan en absoluto la materia del retornable, y corrigen ejercicios mal resueltos como buenos.

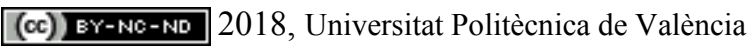


2. Alumnos que dedican poco o incluso nada de tiempo a corregir el retornable y no realizan una correcta verificación de los ejercicios contenidos en él, dándolos todos por buenos.

3. Alumnos que aplican mal los criterios de corrección. Muchos de estos alumnos consideran que el criterio de corrección penaliza excesivamente el error, y deciden aplicar una penalización menor.

Si la nota del alumno es menor que la del profesor es debido a:

1. Alumnos que no han comprendido correctamente alguna parte de la materia relacionada con el retornable y dan por erróneas soluciones correctas. En la mayoría de los casos estos alumnos presentan a su compañero su propia solución, por lo que suele producirse un debate entre ellos o con el profesor.

2. Alumnos que aplican incorrectamente los criterios de corrección penalizando excesivamente respuestas erróneas. Este caso es poco frecuente.

\section{Conclusiones}

En este trabajo se presenta un estudio sobre la fiabilidad de la nota asignada por los alumnos. Esta fiabilidad se define como la concordancia con la nota que asignaría un profesor.

Los datos muestran que para alrededor del $60 \%$ de los casos, la discrepancia entre la nota recibida y la nota merecida es de \pm 1 punto, aunque es cierto que esta desviación es más frecuente en favor del alumno, es decir, el alumno recibe una nota mayor que la merecida. Para aproximadamente un $20 \%$, la nota recibida es entre 1 y 3 puntos más alta que la merecida.

De los resultados obtenidos, y considerando que la nota de los retornables supone un $10 \%$ de la calificación final en la asignatura, los autores consideran que la nota de los alumnos puede ser utilizada directamente en la calificación final. La existencia de otros actos de evaluación, presenciales e individuales, ayudará a corregir las desviaciones introducidas por la evaluación por pares.

Para la tranquilidad de los alumnos y de los propios profesores, es importante realizar un muestreo y revisar algunas correcciones, especialmente de aquellos retornables que obtienen la máxima nota, o en el caso de alumnos con nota muy dispar respecto a la de sus anteriores retornables.

Cómo trabajo futuro se pretender identificar los factores que producen errores significativos en la corrección de los alumnos, permitiendo al profesor estar atento y centrar sus esfuerzos de revisión en los casos importantes. 


\section{Referencias}

DAVIES, R., and BERROW, T. (1998), “An evaluation of the use of computer sopported peer review for developing higer-level skills". Computers \& Education. Volume 30, Issues 1-2, January 1998, Pages 111-115

IBARRA, M.S., RODRIGUEZ, G., y GOMEZ, M. A. (2012) "La evaluacion entre iguales;beneficios y estrategias para su práctica en la universidad". Revista de la Educación. 359, septiembre-diciembre 2012, pp206-231.

Krathwohl, R.R. , Bloom, B.S., and MasiA, B. (1994). Taxonomy of Educational Objectives: The Classification of Educational Goals. Handbook 2: Affective Domain. London, UK. Longman July.

MARQUES, M., BADIA, J. M., MARTINEZ-MARTIN, E.(2013) "Una experiencia de evaluación formadora por compañeros" . ReVisión, Vol 6, No 2, 2013. http://hdl.handle.net/10234/84749 Accedido 1 de marzo de 2018

MARTÍ-CAMPOY, A., ALONSO, M., PETIT MARTÍ, S., ATIENZA VANACLOIG, V., MARTÍNEZ, J. C., GONZÁLEZ, A. (2012) "Los retornables como herramienta motivadora en Fundamentos de computadores". En XXI Congreso Universitario de Innovación Educativa en las Enseñanzas Técnicas (CUIEET). Valencia, 2013. Pp 1009-1020.

MARTÍ-CAMPOY, A., PETIT MARTÍ, S., GASSÓ MATOSES, M. T., ATIENZA VANACLOIG, V., RODRÍGUEZ-BALLESTER, F. (2014) "Using peer-assessed returnables in multiple stages to improve learning in computer organization courses". En XI Congreso Bienal de Tecnología, Aprendizaje y Enseñanza de la Electrónica (TAEE). Bilbao, Julio 2014. Pp: 193-198. 